\title{
An optimised clearing protocol for the quantitative assessment of sub-epidermal ovule tissues within whole cereal pistils
}

\author{
Laura G. Wilkinson ${ }^{1}$ and Matthew R. Tucker ${ }^{2 *}$ (i)
}

\begin{abstract}
Background: Seed development in the angiosperms requires the production of a female gametophyte (embryo sac) within the ovule. Many aspects of female reproductive development in cereal crops are yet to be described, largely due to the technical difficulty in obtaining phenotypic information at the cellular or sub-cellular level. Hoyer's solution is currently well established as a solution for clearing thin tissues samples, such as sections or whole tissues of bryophytes, mycorrhizal fungi, and small model organisms (e.g. Arabidopsis thaliana).

Results: Here we report a Hoyer's solution-based clearing method to facilitate clearing of the whole barley pistil, with high reproducibility. The clearing process takes 10 days from fixation to visualisation, whereupon tissue is sufficiently clear to obtain multiple phenotypic measurements from sub-epidermal tissues and cells within the ovule.

Conclusion: Visualisation of cereal ovules that have not been dissected from the pistil allows an unprecedented capability to collect quantitative morphological information from the developing ovule, integument, nucellus and embryo sac. This will enable comparisons with genetic data to reveal the contribution of pre-fertilisation ovule tissues towards downstream seed development.
\end{abstract}

Keywords: Microscopy, Hoyer's solution, Barley, Ovule, Monocot, Development, Cereal, Clearing

\section{Background}

Sustaining food production above the level of food demand is a growing global challenge. Estimates suggest that crop yields will need to increase by $25-75 \%$ to ensure sufficient food production for the world's population in 2050 [1]. Cereal crop production is highly reliant upon development of flowers. In particular, the single ovule within each flower is essential, as it is the site of gametogenesis, fertilisation and downstream grain development. Environmental events such as drought, high temperatures and frost are known to disrupt flower and seed development, causing a reduction in both grain number and grain quality, thus compromising yield [2-4].

\footnotetext{
*Correspondence: matthew.tucker@adelaide.edu.au

${ }^{2}$ School of Agriculture, Food and Wine, University of Adelaide, Waite

Campus, Urrbrae, SA, Australia

Full list of author information is available at the end of the article
}

Our understanding of floral development and seed formation in flowering plants has been dramatically expanded by research in diverse model dicots, such as Arabidopsis thaliana, Hieracium sp., and Torenia fournieri [5-7]. The formation of ovule primordia, the differentiation of a megaspore mother cell from somatic precursors and the production and fertilisation of an embryo sac have been described in intimate molecular, genetic and morphological detail [8]. Research in rice, maize, wheat and barley has contributed significant molecular and genetic knowledge of monocot inflorescence and flower development [9-12]. Despite this, remarkably litthe is known about ovule development in these important cereal species, particularly in regards to how different tissues contribute to eventual seed size, composition and shape. Studies have shown that ovary size is an important component of floret and grain survival [13], but the contribution of constituent tissues remains unclear. Determining the role of these tissues for downstream seed 
development requires robust, high throughput methods for quantitative two and three-dimensional analysis of developing ovule tissues, such that phenotypic information can be extracted and assessed.

Observation of the internal morphology of cleared floral organs is a powerful tool that allows examination of phenotypic alterations in internal structures following genetic or environmental modification, without the need for thin-sectioning. Chemical treatment to clear small tissue samples is a well-established practice, with reagents ranging from the more traditional methyl salicylate, lactic acid and chloral hydrate based solutions [14-16] to recently developed methods such as ClearSee $[17,18]$ and PEA-CLARITY [19]. Despite this, observation of female reproductive tissues in cereal monocots remains technically challenging, contributing to a lack of specific genetic and mechanistic information about gametogenesis and ovule development. Two key technical challenges include the relatively large size of the pistils, which are sufficiently thick to remain opaque when treated using previously published clearing protocols designed for substantially smaller tissues (e.g. [20]), and the ease by which the physical structure of the ovule may be damaged during the process of dissection.

Here we report a robust method for clearing whole cereal pistils with Hoyer's Solution [14], allowing visualisation of wheat and barley ovule ultrastructure in a manner that preserves the physical integrity of internal structures. Experimental variation of incubation time offers flexibility in sample preparation, yielding exceptionally clear tissue after a minimum of 10 days post tissue collection and up to a maximum of 16 weeks. The utility of the method was demonstrated by using optical sections through cleared pistils to measure the dimensions of component tissues, enabling phenotypic variation in ovule development to be captured within a panel of barley cultivars.

\section{Methods}

Reagents

Chloral Hydrate C-IV (\#15307, Sigma-Aldrich, Australia)

Ethanol (\#EA043-2.5L, Chem-Supply, Australia)

Formaldehyde (\#809, Ajax Finechem, Australia)

Glacial Acetic Acid (\#2335, Ajax Finechem, Australia)

Glycerol (\#242, Ajax Finechem, Australia)

\section{Solutions}

FAA fixative [21] 50\% Ethanol (v/v), 10\% Formaldehyde ( $37 \%$ solution, also called formalin), $5 \%$ glacial acetic acid $(\mathrm{v} / \mathrm{v})$, and $35 \%$ sterile water $(\mathrm{v} / \mathrm{v})$.
Ethanol Series 100\% analytical grade EtOH diluted in water to a concentration of 70,80 and $90 \%$, and $100 \%$ EtOH filtered through a molecular sieve.

Chloral hydrate solution 250 g chloral hydrate dissolved in $100 \mathrm{~mL}$ sterile water

Hoyer's Solution [14] A 3.0:0.8:0.2 mixture of chloral hydrate:water:glycerol.

\section{Equipment}

Greenhouse facility

Standard laboratory $4{ }^{\circ} \mathrm{C}$ refrigerator

Fume cupboard

Compound microscope with differential contrast (DIC) and Nomarski filter for a $\times 10, \times 20$ and/or $\times 40$ objective

Computer and free ZEN 2011 Blue (Zeiss) LE software Ventilated microscopy slide box

Small exhaust fan

Glass pipettes

Fine point tweezers (Dumont \#5, Emgrid, Australia)

Liquid scintillation vials (\#Z190535, SigmaAldrich, Australia)

Polysine Slides (\#P4981, ThermoFisher Scientific, Australia)

$22 \times 40 \mathrm{~mm}$ Cover slips (\#G422, ProSciTech, Australia)

Microflex 93-260 chemical resistant gloves (Ansell, Australia)

\section{Plant growth and staging}

Barley plants were grown in greenhouse facilities at The Plant Accelerator (Adelaide, Australia), under $22^{\circ} \mathrm{C}$ (day) and $17{ }^{\circ} \mathrm{C}$ (night) temperatures without addition of supplemental light (Fig. 1a). Florets were identified to be at anthesis by removing them from spikes (Fig. 1b), gently reaching inside the palea and lemma with tweezers then assessing the colour of the anthers and how readily pollen was released upon gentle squashing. At anthesis, the anthers are a rich yellow colour and have yet to shed pollen, but readily release pollen with minimal application of pressure when pressed against a thumbnail (Fig. 1c). Any florets that contained green or green/yellow anthers, or anthers that had already shed pollen, were discarded.

\section{Sample collection and fixation (timing: $10 \mathrm{~min}$ per tiller + overnight fixation)}

Whole pistils were removed from anthesis barley flowers by reaching inside the flower with fine tweezers and pinching the base of the pistil as low as possible (Fig. 1c). Care was taken to avoid tearing the base of the pistil where the ovule is located. Lodicules were gently removed from the outside of the pistil before placing it in 


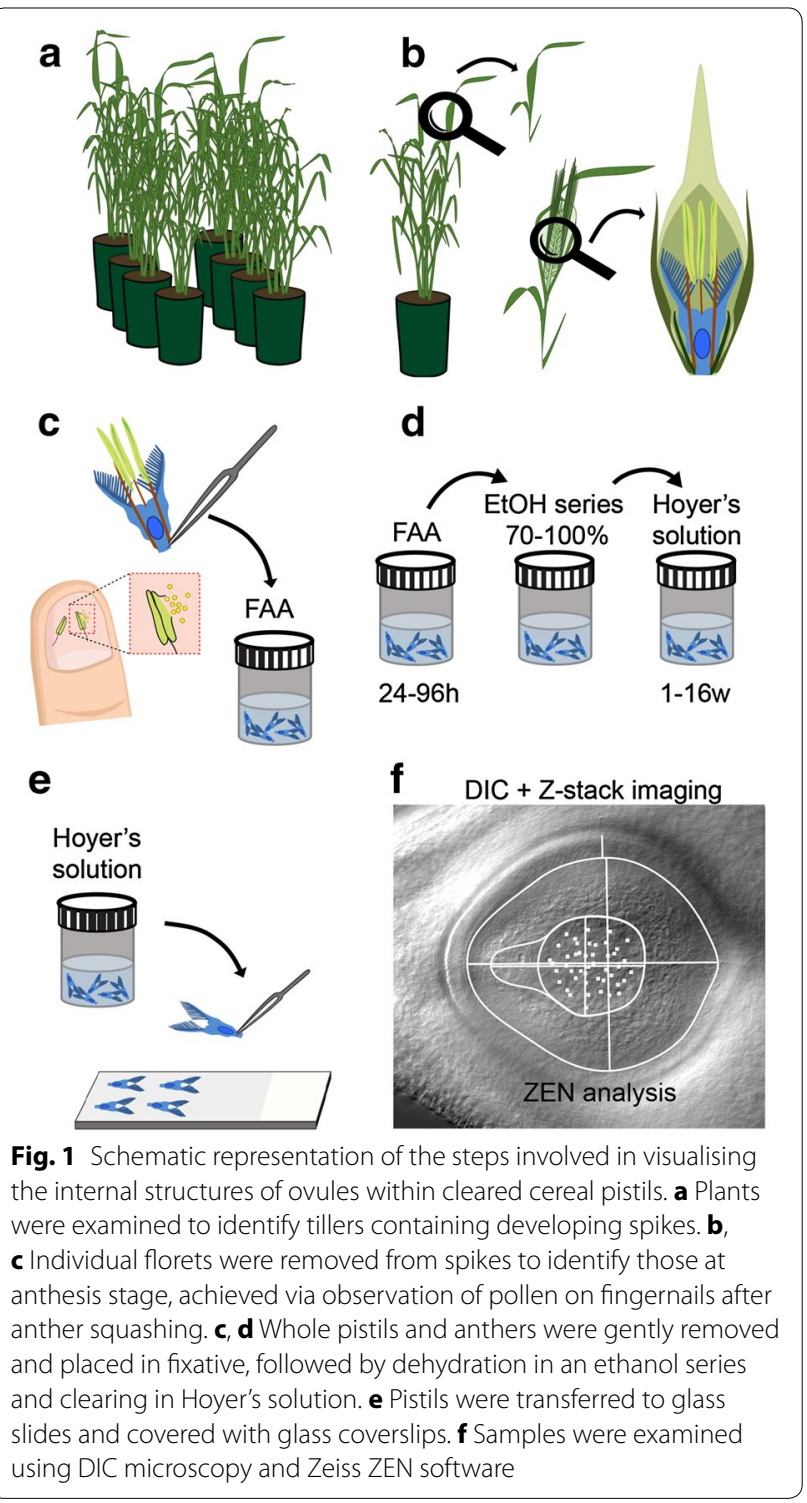

a flat bottomed glass scintillation vial containing $2 \mathrm{~mL}$ of ice cold FAA fixative.

\section{Sample dehydration (timing: $4 \mathrm{~h}+$ overnight dehydration $+\mathbf{4}$ to 120 days incubation)}

Within 1 week of fixation barley pistils were dehydrated through an ethanol series and placed into Hoyer's solution (Fig. 1d), using fine-tipped glass pipettes for each fluid exchange to minimise the possibility of damage to tissue samples. The $\mathrm{EtOH}$ series comprised of $3 \times 20 \mathrm{~min}$ washes at 70, 80, 90 and $100 \% \mathrm{EtOH}$ at room temperature. Samples were left in the final $100 \% \mathrm{EtOH}$ wash overnight before transfer into $4 \mathrm{~mL}$ Hoyer's Solution. Samples must remain immersed in Hoyer's solution at room temperature for a minimum of 4 days.
Long protocol Samples may remain gently infiltrating in Hoyer's solution for up to 16 weeks. Incubation for 4 weeks preserves tissue quality ideally for imaging of embryo sac features. Vials must be tightly sealed if samples are to be stored for longer than 2 weeks.

\section{Sample mounting (timing: 15 min per slide +2 to 4 days incubation)}

Pistil tissues were manipulated with fine point tweezers and only held by the stigma in order to avoid crushing the ovary wall, ovule or surrounding tissue. Pistils were placed on flat Poly-Lys coated glass microscopy slides with either the dorsal or ventral side down so that both stigma of each pistil lay "flat", rather than one stigma pointing up into the air (Fig. 1e). On each slide, pistils were placed equidistantly in a symmetrical arrangement and gently covered with a $22 \times 40 \mathrm{~mm}$ coverslip. This arrangement allows the pistils to lie flat, ensures that variation in the relative viewing angle of the ovule is limited, and preserves the structural integrity of the ovule by preventing any damage to the tissue. Following sample arrangement and application of the cover slip, Hoyer's Solution was pipetted underneath the cover slip onto the slide until all air was evacuated. Slides were then placed flat into a slide storage box that allowed limited ventilation and left in a fume cupboard for 4 days. Samples stored in a well ventilated location are cleared in 24-48 h depending upon the degree of ventilation. Conversely, samples stored after mounting with insufficient or no ventilation required up to 14 days to clear sufficiently to allow visualisation. Therefore, the degree of ventilation can be used to tailor the method to suit the user's time constraints.

Long protocol Samples incubated in $4 \mathrm{~mL}$ Hoyer's Solution for longer than 2 weeks typically require less than 4 days to clear completely once mounted on the microscopy slide. For example, tissue stored in Hoyer's Solution for 8-16 weeks generally does not require a period of ventilated storage longer than $12 \mathrm{~h}$, and in some cases may be visualised immediately after mounting on slides.

\section{Imaging (timing: 2 min per piece of tissue)}

Pistils were imaged using differential contrast microscopy (DIC) at $\times 10$ magnification with a Zeiss AxioImager M2 equipped with a Nomarski filter. For comprehensive data collection, optical slices spanning from the dorsal to ventral integument were taken as a $z$-stack image, using Zeiss ZEN 2011 (Blue) software. 


\section{Image analysis (timing: 10 to 15 min per image)}

Data were analysed using the Zeiss ZEN 2011 (Blue) software package. Diverse measurements were taken including the 2-dimensional area $\left(\mu \mathrm{m}^{2}\right)$ of each ovule tissue of interest, using the "contour (spline)" graphics tool to encircle the tissue, as well as the longitudinal and transverse dimensions $(\mu \mathrm{m})$ of the same tissues, using the "line" graphics tool, and the antipodal nuclei were counted using the "event marker" graphics tool (Fig. 4c). Measurements were taken by following tissue boundaries for each given trait throughout optical sections and placing contour markers at the widest point. Two-dimensional ovule area was measured at the boundary between integument and nucellus. Embryo sac area was measured by tracing the outline of the structure from the micropyle to the chalazal region. The residual somatic cell (nucellus) area was measured by subtracting the embryo sac area from the whole ovule area.

\section{Results}

\section{Protocol timing optimisation}

Clearing was most successful when fixative was removed through an ethanol dehydration series prior to a 4-day infiltration step in Hoyer's solution, followed by a 4-day rest after mounting on microscopy slides (Fig. 2a). Equally clear images were obtained from samples that were dehydrated, left to gently infiltrate in Hoyer's solution for 4 weeks, then imaged directly after mounting on microscopy slides (Additional file 1: Fig S1A). The maximum period of incubation that achieved acceptable clearing was approximately 16 weeks (Additional file 1: Fig S1B). Deterioration of cellular morphology was seen when samples were left in scintillation vials to gently infiltrate with Hoyer's solution for longer than 5 months (Fig. 2b), or when samples were mounted on microscopy slides and stored in a well ventilated area for multiple days, or were imaged after 10 days in a semi-ventilated storage box (Additional file 1: Fig S1C). Evaporation of the Hoyer's solution was also a factor that prevented acquisition of acceptable images if the samples were over-ventilated.

\section{Protocol reagent optimisation}

Clearing was not successful when ethanol dehydration was omitted and fixed samples were placed directly in Hoyer's solution (Fig. 2c). Similarly, it was found that use of pure chloral hydrate solution rather than Hoyer's solution yields unacceptably murky images (Fig. 2d), a factor of both the harsher degradation process when chloral hydrate is used in isolation and the lack of glycerol lowering the refractive index of the mounting fluid. Rough sample collection or handling of tissue throughout the dehydration

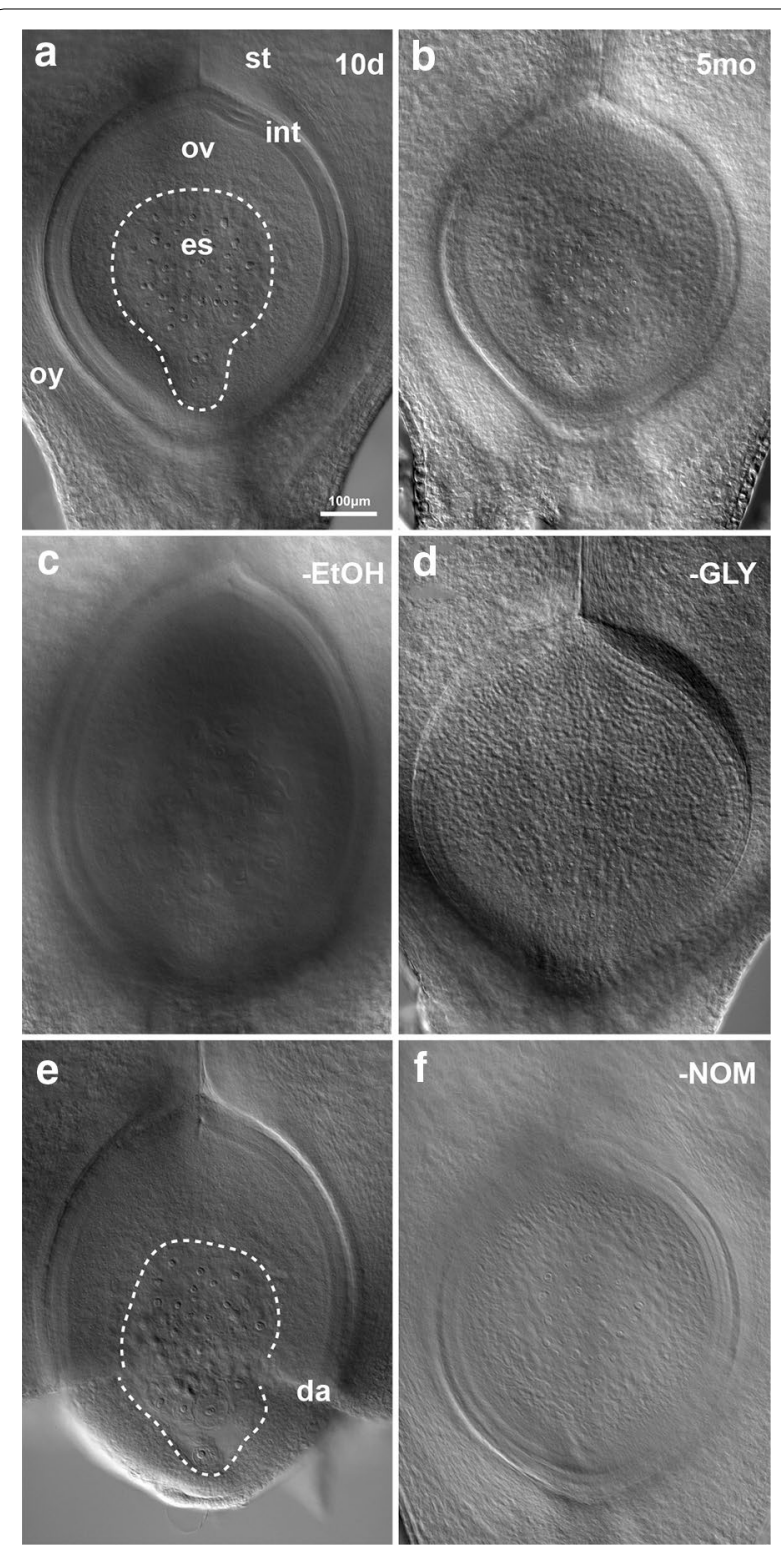

Fig. 2 Barley ovules imaged at $\times 10$ showing the outcomes of variations to the clearing protocol. Images presented as composites, generated by merging optical sections. a A 10-day (10d) method incorporating ethanol dehydration prior to a 4-day infiltration with Hoyer's solution, then a 4-day rest after mounting on microscopy slides produced the greatest clarity of results within a reasonably short time frame. es = embryo sac, ov = ovule, oy = ovary wall, st $=$ style, int $=$ integuments. $\mathbf{b}$ Samples gently infiltrated with Hoyer's solution for over 5 months (5mo) deteriorated, resulting in unacceptably murky images. c Omitting ethanol (-EtOH) dehydration prior to incubation in Hoyer's solution results in the tissue becoming grainy and unacceptably murky. d Incubation of the sample in chloral hydrate without glycerol (-GLY) after fixation and dehydration results in the tissue becoming unacceptably murky. e Rough sample collection and careless handling of the tissues results in damaged ovaries, which may disrupt the internal morphology of the ovule. da = damaged region. $\mathbf{f}$ Samples cannot be imaged without a Nomarski filter $(-\mathrm{NOM})$ 
process often resulted in structural disruption of the sample (Fig. 2e; Additional file 1: Fig. S1D). In addition, a Nomarski filter is essential for image acquisition (Fig. 2f).

\section{Optimised method results}

Cleared pistils offer an excellent opportunity to visualise internal components of the ovule in their native spatial arrangement using a DIC microscope with a Nomarski filter (Figs. 3, 4). Imaging the entire ovule within the ovary is easily possible at $\times 10$ magnification, and is particularly powerful when captured in a series of optical sections, allowing construction of composite images and videos that represent all internal features of the ovule's cellular arrangement (Fig. 3a; Additional file 2: Fig. S2) and measurement of some three-dimensional features such as embryo sac depth. At $\times 40$ magnification, intimate cellular details of the embryo sac and other ovule components could be obtained (Fig. 3b, c), such as clear, prominent nuclei in the egg cell, central cell and antipodal cells. The quality of tissue resolution was similar in pistils that were infiltrated in Hoyer's solution for 10 days (Fig. 3d) and 10 weeks (Fig. 3b).
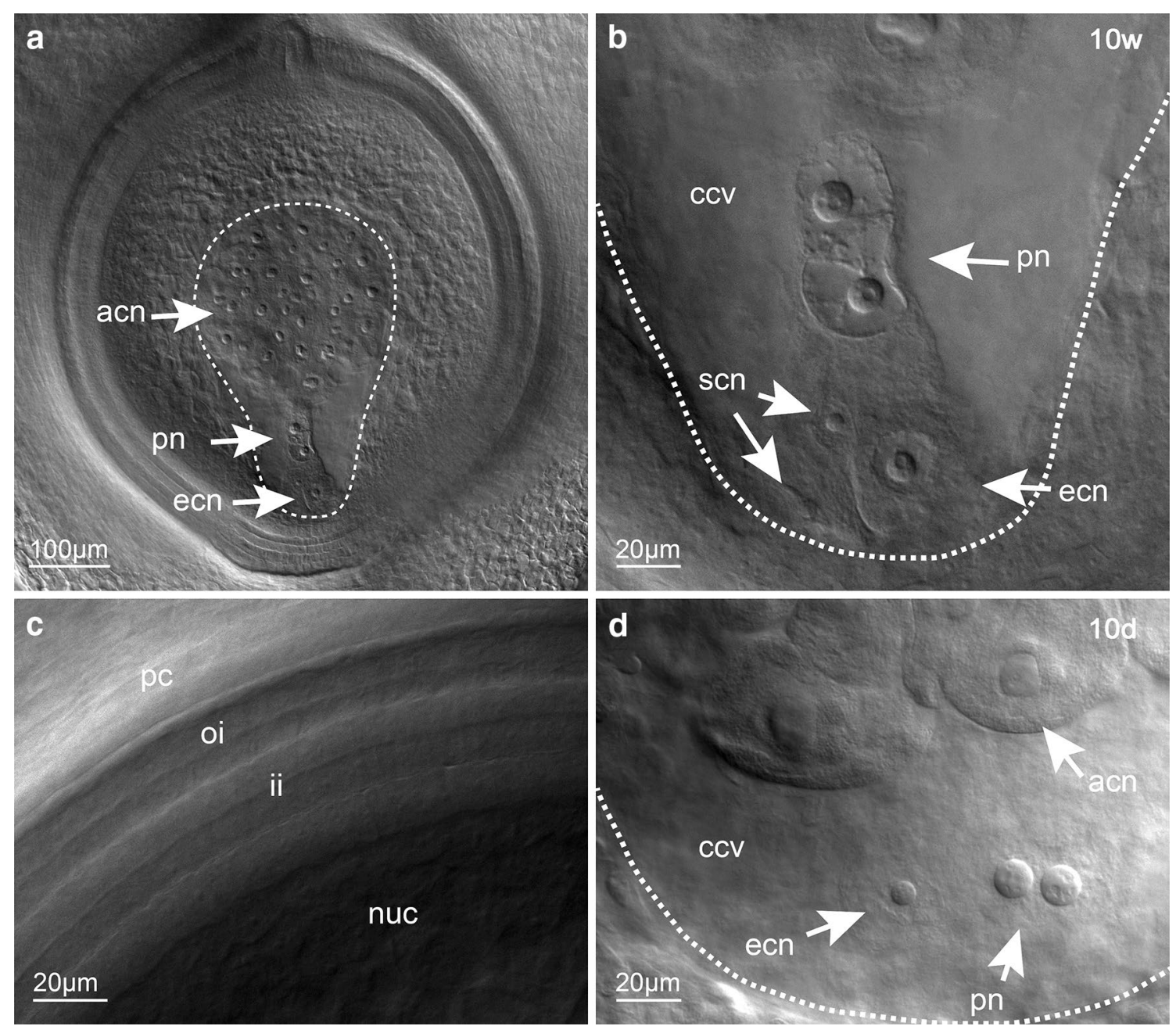

Fig. 3 Structural details of the ovule are visible in cleared barley pistils (H. vulgare cv. Host). Images presented as composites, generated by merging optical sections. a Mature barley ovule imaged at $\times 10$ magnification without dissection from the pistil. b-d Cellular resolution may be achieved with a $\times 40$ objective, allowing clear visualisation of the egg cell nucleus, synergid nuclei, polar nuclei and antipodal cell nuclei using the "long method" i.e. incubation for at least 10 weeks (10w) in Hoyer's solution (b). The integument layers are also visible (c). Similar cellular resolution may also be achieved in samples processed with a 10-day (10d) "short" method (d). acn = antipodal cell nuclei, $c c v=$ central cell vacuole, ecn = egg cell nucleus, $\mathrm{ii}=$ inner integument, $\mathrm{nuc}=$ nucellus, oi $=$ outer integument, $\mathrm{pn}=$ polar nuclei, $\mathrm{pc}=$ pericarp, $\mathrm{scn}=$ synergid cell nuclei. The embryo sac is indicated by a dashed white line 

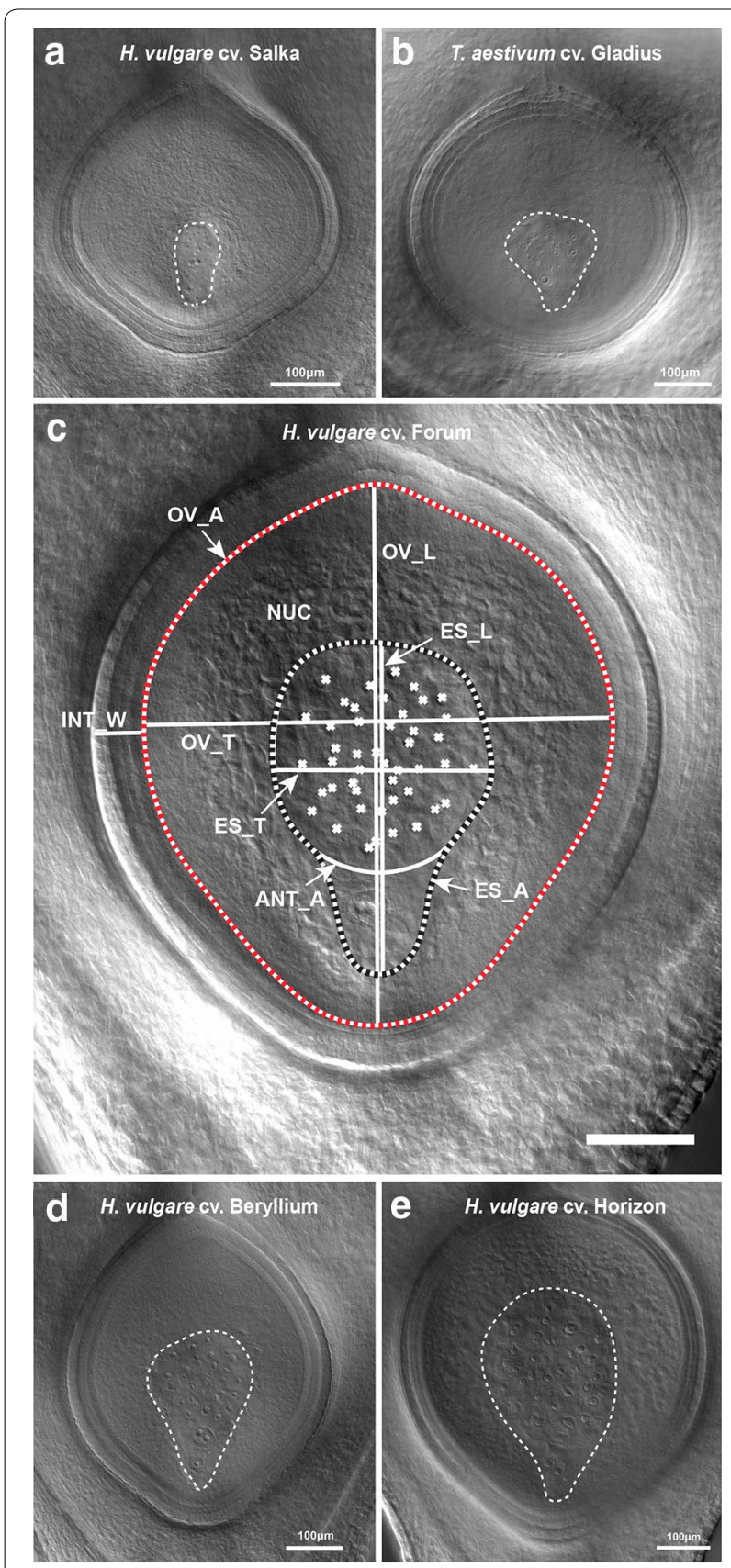

Fig. 4 Ovule morphology after clearing. Ovule images are presented as composites, generated by merging optical sections. The method worked equally well for barley (a) and wheat (b) pistils at various stages. Pre-anthesis ovules are shown. c Measurement of traits at anthesis, including ovule area (OV_A), embryo sac area (ES_A), ovule and embryo sac transverse and longitudinal dimensions (OV_L, OV_T, ES_L,ES_T), integument width (INT_W), antipodal number (marked with crosses) and antipodal cluster area (ANT_A), using Zeiss ZEN software. The ovule area is indicated with a dashed red and white line, while the embryo sac area is indicated by a dashed black and white line. NUC = nucellus. Small (d) and large (e) mature barley ovules after clearing imaged at $\times 10$ magnification without dissection from the pistil. Bar $100 \mu \mathrm{m}$

\section{Sup-epidermal details of ovule development differ between cultivars}

A fundamental understanding of reproductive organ development in cereals ultimately aims to support breeding programs in generating high-yielding, high-quality cultivars. To demonstrate the utility of this clearing technique, we examined pistils from barley and wheat (Fig. 4a, b). In both species, sub-epidermal details of ovule tissues, including the embryo sac, egg cell, central cell, antipodals, integument and nucellus could be discerned and measured (Fig. 4c). To determine if intraspecific differences in ovule development could be identified, we examined a selection of 2-row spring barley cultivars. Quantification of morphological features such as tissue area, thickness and cell number in nine cultivars revealed natural variation in most traits (Table 1; Figs. 4d, e, 5). For example, ovule area in $H$. vulgare cv Horizon was almost twofold larger than $H$. vulgare cv Beryllium (Figs. 4d, e, 5a), antipodal number was lowest in $\mathrm{H}$. vulgare $\mathrm{cv}$. Toucan $(\sim 32 \pm 5)$ compared to $H$. vulgare $\mathrm{cv}$. Horizon $(\sim 49 \pm 4)$ and integument width was thickest in $H$. vulgare cv Agenda $(\sim 50 \pm 4 \mu \mathrm{m})$ and thinnest in $H$. vulgare cv Rainbow ( $41 \pm 3 \mu \mathrm{m})$. Correlation analysis indicated that multiple traits showed strong positive correlations, such as ovule area, embryo sac area and ovule height (Fig. 5b, c), suggesting that these features are intimately related. However, other traits showed weak or no correlations with other ovule features, including integument width, antipodal number, nucellus area and ovule transverse width (Fig. 5b, c).

\section{Discussion}

In this study a method for clearing tissue using Hoyer's solution has been designed to suit cereal pistils such that internal structures of the ovule may be imaged with a high degree of clarity. Chloral hydrate-based clearing solutions have been successfully used in a wide range of biological fields [14, 22, 23], permitting a great deal of fundamental morphological and phenotypic information to be gathered. However, in our hands, previously reported protocols incorporating chloral hydrate that work well in Arabidopsis (e.g. [20, 24]) did not result in sufficient clearing of barley pistils to enable quantitative measurement of individual ovule tissues. Moreover, alternative methods that incorporate methyl salicylate [16, 25-27], lactic acid [28], sodium hypochlorite [29] or sodium hydroxide [30], lack the convenience and/or efficiency of our established Arabidopsis chloral hydratebased method [20]. Other recently reported clearing reagents such as ClearSEE [17], PEA-CLARITY [19] and FocusClear [31] are designed to clear tissue while preserving fluorescent labelling, but are either too expensive 
Table 1 Phenotypic measurements of ovule tissues from nine $\boldsymbol{H}$. vulgare cultivars

\begin{tabular}{|c|c|c|c|c|c|c|c|c|c|c|c|c|}
\hline \multirow[t]{2}{*}{ Cultivar } & \multirow[t]{2}{*}{$\mathbf{n}$} & \multicolumn{3}{|l|}{ Ovule } & \multicolumn{3}{|l|}{ Embryo sac } & \multirow{2}{*}{$\begin{array}{l}\text { Integument } \\
\text { Width }(\mu \mathrm{m})\end{array}$} & \multicolumn{2}{|l|}{ Nucellus } & \multicolumn{2}{|c|}{ Antipodal } \\
\hline & & Area $\left(\mu \mathrm{m}^{2}\right)$ & Trans $(\mu \mathrm{m})$ & Long $(\mu \mathrm{m})$ & Area $\left(\mu \mathrm{m}^{2}\right)$ & Trans $(\mu \mathrm{m})$ & Long $(\mu \mathrm{m})$ & & Area $\left(\mu \mathrm{m}^{2}\right)$ & $\%$ & \# & Area $\left(\mu \mathrm{m}^{2}\right)$ \\
\hline Beryllium & 6 & $138,197.8$ & 396.9 & 484.3 & $30,462.7$ & 174.6 & 270.1 & 44.9 & $107,735.1$ & 78.1 & 37.7 & $25,829.6$ \\
\hline STDEV & & $15,888.7$ & 28.7 & 21.7 & 5597.7 & 26.0 & 25.5 & 5.7 & $11,329.2$ & 2.2 & 2.7 & 5562.9 \\
\hline Novello & 12 & $154,239.1$ & 413.1 & 505.6 & $33,330.3$ & 171.7 & 298.0 & 43.9 & $120,908.9$ & 78.5 & 35.0 & $27,401.0$ \\
\hline STDEV & & $16,522.7$ & 19.1 & 42.8 & 6097.1 & 16.7 & 37.4 & 3.2 & $12,418.2$ & 2.5 & 4.7 & 6447.3 \\
\hline Orbit & 6 & $161,883.0$ & 451.7 & 487.3 & $33,816.5$ & 182.2 & 281.0 & 46.4 & $128,066.6$ & 78.9 & 36.5 & $28,026.6$ \\
\hline STDEV & & $20,556.0$ & 32.4 & 20.9 & 4352.4 & 17.3 & 10.6 & 3.0 & $18,494.2$ & 2.6 & 6.6 & 4934.9 \\
\hline Extract & 11 & $164,652.1$ & 448.3 & 497.6 & $34,479.2$ & 188.9 & 284.0 & 46.7 & $130,172.9$ & 79.1 & 36.0 & $29,457.8$ \\
\hline STDEV & & $14,406.3$ & 22.8 & 21.5 & 7094.9 & 25.8 & 27.4 & 2.7 & $11,537.1$ & 3.5 & 2.9 & 7992.9 \\
\hline Toucan & 11 & $177,561.6$ & 444.7 & 554.2 & $45,330.7$ & 210.7 & 320.3 & 46.1 & $132,230.9$ & 74.7 & 32.6 & $42,617.2$ \\
\hline STDEV & & $21,683.1$ & 25.0 & 44.8 & 9374.7 & 22.8 & 46.4 & 2.1 & $14,255.4$ & 3.3 & 4.7 & 5469.6 \\
\hline Saloon & 13 & $199,385.6$ & 456.7 & 598.7 & $70,066.1$ & 266.1 & 382.3 & 43.6 & $129,319.5$ & 64.9 & 41.8 & $65,125.7$ \\
\hline STDEV & & $11,367.7$ & 19.1 & 25.6 & 9071.9 & 21.7 & 25.0 & 1.7 & $10,869.6$ & 3.9 & 4.8 & 8283.8 \\
\hline Rainbow & 6 & $221,620.1$ & 488.6 & 598.6 & $65,471.9$ & 242.4 & 375.2 & 41.0 & $156,148.2$ & 70.6 & 36.8 & $57,424.8$ \\
\hline STDEV & & $14,500.2$ & 20.3 & 19.8 & 8707.8 & 16.8 & 23.0 & 3.0 & 8030.2 & 2.5 & 3.5 & 7982.3 \\
\hline Agenda & 6 & $227,499.2$ & 483.4 & 625.2 & $75,121.6$ & 272.9 & 367.9 & 49.5 & $152,377.7$ & 66.7 & 45.2 & $72,000.8$ \\
\hline STDEV & & $42,467.0$ & 42.0 & 60.6 & $13,856.8$ & 28.5 & 53.2 & 4.9 & $32,315.5$ & 4.3 & 8.5 & $14,718.3$ \\
\hline Horizon & 6 & $241,310.7$ & 519.2 & 607.2 & $72,158.9$ & 268.8 & 371.9 & 43.0 & $169,151.8$ & 70.0 & 48.8 & $66,487.6$ \\
\hline STDEV & & $48,703.9$ & 48.0 & 59.0 & $14,691.2$ & 29.0 & 48.1 & 1.5 & $35,130.8$ & 2.2 & 3.6 & $14,851.0$ \\
\hline
\end{tabular}

Trans transverse width, Long longitudinal height

for high-throughput analysis or provide insufficient cellular resolution without additional staining.

Although the chloral hydrate-based method we describe is not compatible with visualisation of fluorescently-tagged proteins, it can be applied to diverse cereals, allows customisable incubation times, requires minimal tissue handling, and consistently provided excellent clearing and an ability to detect quantitative differences in tissue development in unstained cereal ovary samples. The Zeiss ZEN software used for image analysis is freely available for download and easy to use, while the FIJI software suite was used to extract similar results [32]. In our pilot study of barley ovules at anthesis, 75 pistils were examined from 9 cultivars. The method was not specifically tested on a microscope containing a motorised 8-slide mounting frame or image stitching software, but such an approach would almost certainly be compatible, suggesting that image acquisition might be automated in future to allow for high-throughput data collection. Whether the scale of analysis required for germplasm screens in breeding populations can be achieved is currently unclear. However, the method is compatible with pre-breeding efforts to dissect pre-fertilisation traits that contribute to downstream seed development and morphology. Furthermore, we anticipate that the method will be particularly useful for the rapid characterisation of mutant phenotypes and transgenic plants that effect ovule development in barley and wheat.

\section{Conclusions}

A clearing technique typically used in the analysis of tissues from dicot model organisms was successfully adapted to clear the much larger cereal pistil. This paves the way for further interrogation of sup-epidermal features of ovule development in barley and other cereal crop species. The application of this method to a large panel of genetically distinct or genetically modified cereal varieties may assist the identification of novel genes controlling ovule phenotypes as well as components of seed yield and quality. 


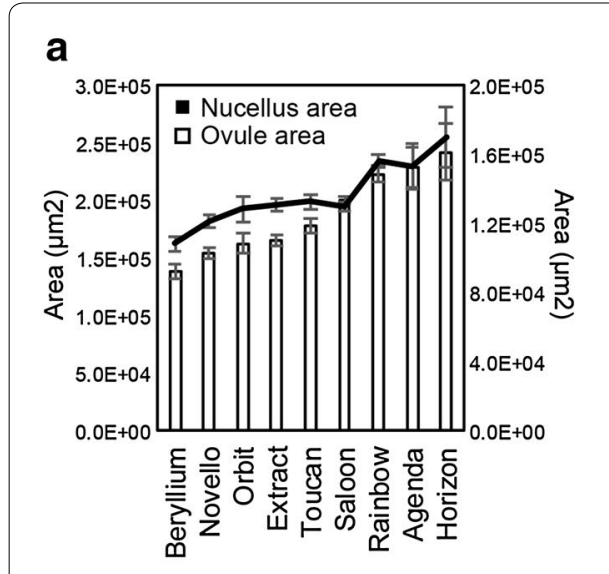

b
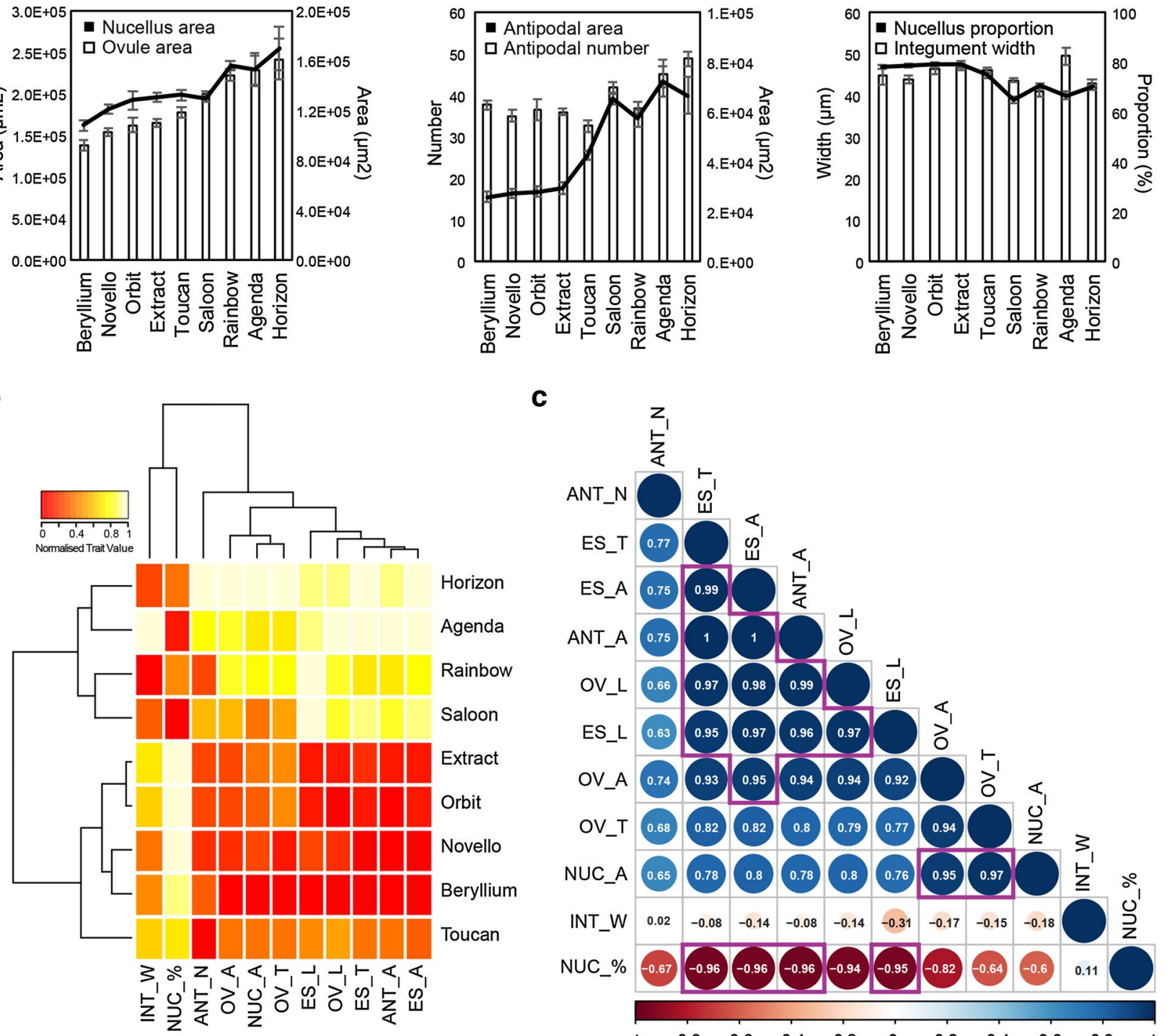

C

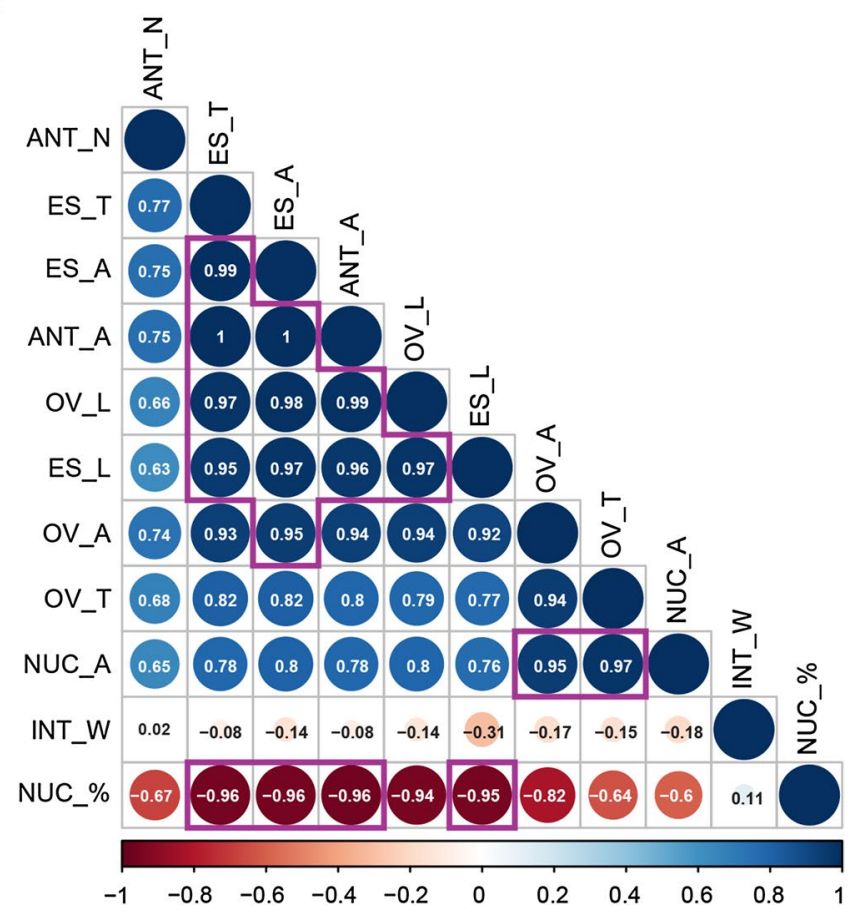

Fig. 5 Analysis of barley ovule traits by pistil clearing. a Ovule phenotypes were examined in nine cultivars of 2-row spring barley. Traits such as ovule area, nucellus area, antipodal number, antipodal area, nucellus proportion and integument width (see Fig. 4) were compared between the cultivars. Error bars show standard error. b Heat map showing the normalised trait values (between 0 and 1) for 11 ovule traits in the 9 examined cultivars. Cultivars and traits were clustered via hierarchical clustering. c Correlation analysis of 11 different ovule traits. The size and colour of the circles indicates the degree of trait correlation, which is also indicated via a numerical value. R-squared values greater than 0.95 are indicated via purple boxes. INT_W = integument width, NUC_\% = nucellus proportion, ANT_N = antipodal number, OV_A = ovule area, NUC_A = nucellus area, OV_T = ovule transverse width, ES_L = embryo sac longitudinal height, OV_L = ovule longitudinal height, ES_T = embryo sac transverse width, ANT_A = entire antipodal area, ES_A = embryo sac area 


\section{Additional files}

Additional file 1: Fig. S1. Barley ovules imaged at $\times 10$ showing the outcomes of variations to the clearing protocol. Images presented as composites, generated by merging optical sections. A Ethanol dehydration prior to a 4-week (4w) gentle infiltration with Hoyer's solution, then imaging samples directly after mounting on microscopy slides produced high clarity results in a longer time frame. B Samples gently infiltrated with Hoyer's solution for 16 weeks ( $16 \mathrm{w}$ ) then immediately imaged produced high-quality results. C Samples left mounted on microscope slides in a well ventilated storage box or for too long were not able to be imaged properly due to evaporation (+Evap) of the Hoyer's solution, causing uneven illumination of the sample and in some cases accelerated degradation of the tissue, resulting in an unacceptably grainy image. D Rough sample collection and careless handling of the tissues results in damaged ovaries, which may disrupt the internal morphology of the ovule. The embryo sac is indicated by a dashed white line.

Additional file 2: Fig. S2. Sequential $2.4 \mu \mathrm{m}$ optical slices $(n=50)$ of a cleared $H$. vulgare cv. Gant ovule at anthesis were combined to generate a movie file.

\section{Authors' contributions}

LGW collected and analysed the data. LGW and MRT designed and tested the method and jointly contributed to writing the manuscript. Both authors read and approved the final manuscript.

\section{Author details}

${ }^{1}$ ARC Centre of Excellence in Plant Cell Walls and School of Agriculture, Food and Wine, University of Adelaide, Waite Campus, Urrbrae, SA, Australia.

${ }^{2}$ School of Agriculture, Food and Wine, University of Adelaide, Waite Campus, Urrbrae, SA, Australia.

\section{Acknowledgements}

We wish to thank Rachel Burton and Caitlin Byrt for advice, Ryan Whitford for wheat samples and Plant Accelerator staff for maintaining plants. We also wish to acknowledge members of the Tucker Laboratory and the ARC Centre of Excellence in Plant Cell Walls for useful discussions and suggestions.

\section{Competing interests}

The authors declare that they have no competing interests.

\section{Availability of data and materials}

The datasets used and/or analysed during the current study are available from the corresponding author on reasonable request.

\section{Consent for publication}

All authors give consent for the data to be published.

Ethics approval and consent to participate Not applicable.

\section{Funding}

This work was supported by an Australian Research Council (ARC) (Grant No. FT140100780) Centre of Excellence in Plant Cell Walls supplementary Ph.D. scholarship (LGW) and an ARC Future Fellowship (MRT).

\section{Publisher's Note}

Springer Nature remains neutral with regard to jurisdictional claims in published maps and institutional affiliations.

Received: 29 June 2017 Accepted: 8 August 2017

Published online: 15 August 2017
References

1. Hunter MC, Smith RG, Schipanski ME, Atwood LW, Mortensen DA. Agriculture in 2050: recalibrating targets for sustainable intensification. Bioscience. 2017;67(4):386-91.

2. Onyemaobi I, Liu H, Siddique KHM, Yan G. Both male and female malfunction contributes to yield reduction under water stress during meiosis in bread wheat. Front Plant Sci. 2016;7:2071.

3. Saini HS, Westgate ME. Reproductive development in grain crops during drought. Adv Agron. 1999;68:59-96.

4. Thakur P, Kumar S, Malik JA, Berger JD, Nayyar H. Cold stress effects on reproductive development in grain crops: an overview. Environ Exp Bot. 2010;67(3):429-43.

5. Susaki D, Takeuchi H, Tsutsui H, Kurihara D, Higashiyama T. Live imaging and laser disruption reveal the dynamics and cell-cell communication during Torenia fournieri female gametophyte development. Plant Cell Physiol. 2015;56(5):1031-41.

6. Tucker MR, Koltunow AMG. Traffic monitors at the cell periphery: the role of cell walls during early female reproductive cell differentiation in plants. Curr Opin Plant Biol. 2014;17:137-45.

7. Yang W-C, Sundaresan V. Genetics of gametophyte biogenesis in Arabidopsis. Curr Opin Plant Biol. 2000;3(1):53-7.

8. Yang W-C, Shi D-Q, Chen Y-H. Female gametophyte development in flowering plants. Annu Rev Plant Biol. 2010;61:89-108.

9. Boden SA, Weiss D, Ross JJ, Davies NW, Trevaskis B, Chandler PM, Swain SM. EARLY FLOWERING3 regulates flowering in spring barley by mediating gibberellin production and FLOWERING LOCUS T expression. Plant Cell. 2014;26(4):1557-69.

10. Yoshida H, Nagato Y. Flower development in rice. J Exp Bot. 2011:62(14):4719-30.

11. Youssef HM, Eggert K, Koppolu R, Alqudah AM, Poursarebani N, Fazeli A, Sakuma S, Tagiri A, Rutten T, Govind G. VRS2 regulates hormone-mediated inflorescence patterning in barley. Nat Genet. 2017;49(1):157-61.

12. Zhang D, Yuan Z. Molecular control of grass inflorescence development. Annu Rev Plant Biol. 2014;65:553-78.

13. Guo Z, Schnurbusch T. Variation of floret fertility in hexaploid wheat revealed by tiller removal. J Exp Bot. 2015;66(19):5945-58.

14. Anderson LE. Hoyer's solution as a rapid permanent mounting medium for bryophytes. Bryologist. 1954;57(3):242-4.

15. Cunningham JL. A miracle mounting fluid for permanent whole-mounts of microfungi. Mycologia. 1972;64(4):906-11.

16. Stelly DM, Peloquin S, Palmer RG, Crane CF. Mayer's hemalum-methyl salicylate: a stain-clearing technique for observations within whole ovules. Stain Technol. 1984;59(3):155-61.

17. Kurihara D, Mizuta Y, Sato Y, Higashiyama T. ClearSee: a rapid optical clearing reagent for whole-plant fluorescence imaging. Development. 2015;142(23):4168-79.

18. Timmers AC. Light microscopy of whole plant organs. J Microsc. 2016;263(2):165-70.

19. Palmer WM, Martin AP, Flynn JR, Reed SL, White RG, Furbank RT, Grof CP. PEA-CLARITY: 3D molecular imaging of whole plant organs. Sci Rep. 2015:5:13492.

20. Tucker MR, Okada T, Hu Y, Scholefield A, Taylor JM, Koltunow AM. Somatic small RNA pathways promote the mitotic events of megagametogenesis during female reproductive development in Arabidopsis. Development. 2012;139(8):1399-404.

21. Young B, Sherwood R, Bashaw E. Cleared-pistil and thick-sectioning techniques for detecting aposporous apomixis in grasses. Can J Bot. 1979;57(15):1668-72.

22. Berleth $\mathrm{T}$, Jurgens $\mathrm{G}$. The role of the monopteros gene in organising the basal body region of the Arabidopsis embryo. Development. 1993; 118(2):575-87.

23. Enugutti B, Schneitz K. Microscopic analysis of Arabidopsis ovules. Flower Dev Methods Protocols. 2014;1110:253-61.

24. Franks RG. Histological analysis of the arabidopsis gynoecium and ovules using chloral hydrate clearing and differential interference contrast light microscopy. Oogenesis Methods Protoc. 2016;1457:1-7.

25. Herr J Jr. Recent advances in clearing techniques for study of ovule and female gametophyte development. Angiosperm Pollen Ovules. 1992;149:154. 
26. Koltunow AM. Apomixis: embryo sacs and embryos formed without meiosis or fertilization in ovules. Plant Cell. 1993;5(10):1425-37.

27. Ponitka A, Ślusarkiewicz-Jarzina A. Cleared-ovule technique used for rapid access to early embryo development in Secale cereale $x$ Zea mays crosses. Acta Biol Crac Ser Bot. 2004;46:133-7.

28. Desfeux C, Clough SJ, Bent AF. Female reproductive tissues are the primary target of agrobacterium-mediated transformation by the arabidopsis floral-dip method. Plant Physiol. 2000;123(3):895-904.

29. Aditya J, Lewis J, Shirley NJ, Tan HT, Henderson M, Fincher GB, Burton RA Mather DE, Tucker MR. The dynamics of cereal cyst nematode infection differ between susceptible and resistant barley cultivars and lead to changes in $(1,3 ; 1,4)$ - $\beta$-glucan levels and HvCsIF gene transcript abundance. New Phytol. 2015;207(1):135-47.
30. Tomer E, Gottreich M, Gazit S. Defective ovules in avocado cultivars. J Am Soc Hortic Sci. 1976;101(5):620-3.

31. Chung K, Wallace J, Kim S-Y, Kalyanasundaram S, Andalman AS, Davidson TJ, Mirzabekov JJ, Zalocusky KA, Mattis J, Denisin AK. Structural and molecular interrogation of intact biological systems. Nature. 2013;497(7449):332-7.

32. Schindelin J, Arganda-Carreras I, Frise E, Kaynig V, Longair M, Pietzsch T, Preibisch S, Rueden C, Saalfeld S, Schmid B. Fiji: an open-source platform for biological-image analysis. Nat Methods. 2012;9(7):676-82.

\section{Submit your next manuscript to BioMed Central and we will help you at every step:}

- We accept pre-submission inquiries

- Our selector tool helps you to find the most relevant journal

- We provide round the clock customer support

- Convenient online submission

- Thorough peer review

- Inclusion in PubMed and all major indexing services

- Maximum visibility for your research

Submit your manuscript at www.biomedcentral com/submit 